\title{
La teoría de los sistemas no lineales: mezcla de azar y determinismo
}

Aprobado: 10 de julio de 2020 Recibido: 2 de marzo de 2020 DOI: http://dx.doi.org/10.18566/rfts.v35n35.a03

\section{Omar Arango Otálvaro}

Sociólogo de la UNAULA, magister en Educación de la $U$ de A, profesor titular del Centro de Humanidades de la UPB. 


\section{Resumen}

La teoría de los sistemas no lineales no sigue estrictamente el "dogma general de la invariabilidad de las leyes físicas" en el análisis del sistema social, toda vez que la realidad es una continuidad vital de orden y caos, contrariamente al sistema cerrado y autosuficiente del análisis funcional, que sigue estrictamente el modelo del reloj en el que lo previsible y determinado forma parte de la realidad física. Sin embargo, la explicación de los hechos pasa por tendencias deterministas y tendencias aleatorias, se logra, en tal sentido, que las predicciones y la probabilidad vayan juntas, dejando en claro que el mundo físico y la realidad social no son determinismo absoluto, ni azar permanente, ni admisión sin reservas que abre las puertas del futuro sobre la posibilidad de nuevos análisis del caos al orden y del orden al caos.

\section{Palabras clave}

Azar, Determinismo, Retroalimentación negativa, Retroalimentación positiva, Energía, Desplazamiento, Punto de bifurcación, Proceso amplificador.

\section{Abstract}

The theory of non-linear systems does not strictly follow the "general dogma of the invariability of physical laws" in the analysis of the social system, since reality is a vital continuity of order and chaos, contrary to the closed and self-sufficient system of the functional analysis, which strictly follows the model of the time clock in which the predictable and determined is part of physical reality.

However, the explanation of the facts goes through deterministic tendencies and random tendencies, achieving that in this sense, predictions and probability go together, making it clear that the physical world and social reality are not absolute determinism, nor permanent chance, nor unreserved admission that opens the doors of the future to the possibility of new analyzes from chaos to order and the other way around.

\section{Keywords}

Chance, Determinism, Negative feedback, Positive feedback, Energy, Displacement, Bifurcation point, Amplifying process. 
La teoría enunciada en el título sostiene que el universo no sigue estrictamente el "dogma general de la invariabilidad de las leyes físicas" (Comte, 1999, p. 81), de forma tal que pueda adelantar o fijar los hechos “confinados a axiomas definidos e inmutables" (Zeitlin, 1977, p. 4), como sí lo ha postulado el análisis funcional en la explicación del suicidio mediante una "ley" sociológica, casi un "acto de Dios". En ese sentido, el sistema cerrado y autosuficiente de Durkheim (1897) (citado por Zeitlin, 1977) deduce que "el suicidio varía en razón inversa al grado de integración de los grupos sociales de los que forma parte el individuo" (p. 307).

El mundo físico presenta aspectos caóticos llenos de incertidumbre e incontrolables, allí no es posible la predicción, "ni las monarquías absolutas". Un ejemplo claro es la perspectiva del principio de indeterminación del electrón de Heisenberg, que establece la imposibilidad de conocer simultáneamente la posición y la velocidad del electrón y, por tanto, la imposibilidad de determinar su trayectoria. En general, el principio de incertidumbre de Heisenberg, supone que la materia es dinámica y no por completo predecible. Pareciera un principio cataclísmico contra la monarquía de la predicción.

El modelo de los aspectos caóticos e irreversibles de sistemas abiertos, no es el único modelo teórico, el paisaje de otro lado, lo continúa el modelo de la teoría determinista o de las leyes eternas. El modelo de los sistemas dinámicos presenta, desde su prisma, antecedentes precursores en un matemático francés, Henri Poincaré y en un meteorólogo y matemático norteamericano llamado Edward Lorenz. El modelo determinista encuentra sus formulaciones, entre otros personajes, en el físico y matemático inglés Isaac Newton y en el continuador de la mecánica newtoniana Pierre-Simon Laplace, astrónomo, físico y matemático francés.

Henri Poincaré había ensayado en 1908 con sistemas matemáticos no lineales, impredecibles y caóticos. Sus conclusiones a futuro, se convertirían en "un importante antecedente histórico y conceptual de la teoría del caos" (Cazau, 2016, p. 67). Por su parte, Edward Lorenz, partiendo de una hipótesis, la del efecto multiplicativo del error que le sugirieron ciertos sistemas matemáticos especiales a Poincaré, es decir, que "un pequeño error en las condiciones iniciales, en vez de provocar también un pequeño error en las últimas, provocaría un error enorme en estas, con lo cual el fenómeno se 
vuelve impredecible y entonces se adjudica al azar" (Cazau, 2016, p. 67), la interpretaría como la imposibilidad de pronosticar fenómenos, es decir, los sistemas matemáticos no lineales encerrarían sistemas caóticos: pequeñas variaciones producen grandes cambios.

Más aún, a comienzos de la década del 60 del siglo anterior, Lorenz se dio a la tarea de elaborar "un modelo matemático para predecir fenómenos atmosféricos, y por casualidad descubrió que la misma herramienta matemática que utilizaba estaba fallando: pequeños cambios en las condiciones iniciales producían diferencias asombrosas, inesperadas, impredecibles, en el resultado" (Cazau, 2016, p. 68), detectando sistemas caóticos en las matemáticas, con lo cual las predicciones meteorológicas a mediano o largo plazo resultaban imposibles. Su obra dio lugar finalmente a la teoría del caos.

Esta es la joya de la corona de la teoría del caos que tendrá en el químico belga Ilya Prigogine su portaestandarte, para quien el azar forma efectivamente parte de la realidad física, mezcla de orden y desorden, de determinismo e indeterminismo. Este movimiento o principio de indeterminación, es el que pretende explicar la teoría de los sistemas dinámicos del azar y de cómo estos llevan a nuevas estructuras disipativas, del mismo modo que explicar bajo qué condiciones se evoluciona de la probabilidad a la ley. A lo anterior se le dedicará un tiempo, un espacio y unas circunstancias.

Para la teoría del caos, conocer la previsión exacta de los fenómenos desde lo investigativo, es poco probable, porque la tendencia evolutiva de los sistemas avanza inestable e irreversiblemente en un presente sin derroteros y al azar, que no se inspira en el arquetipo de leyes eternas enteramente diferentes de la imprevisibilidad. Esto se expresa con claridad meridiana, "en algunos campos, y especialmente en la sociedad y su estructura, donde ya han ocurrido desplazamientos" (Drucker, 1993, p. 25), así, como por ejemplo, en los estudios de la organización social de los sistemas alejados de la reciprocidad de fuerzas de todos los elementos que sirven para estructurar la acción social, que es uno de los distintos campos a explorar en este ejercicio de escritura desde la teoría sociológica de la inestabilidad.

En orden a estas ideas, el análisis de un sistema complejo, abierto, como el social o el sistema general de la acción, depende de un enorme conjunto de circunstancias inciertas, que determinan que cualquier pequeña variación 
en un subsistema de la acción, produzca más temprano que tarde o más tarde, no se sabe -lo que se sabe es que la energía es fundamental para la evolución de los subsistemas dentro del sistema general de la acción-, un efecto fuerte en la termodinámica del sistema de la acción, como si hubiese llegado el fin del mundo.

En estas circunstancias, el modelo sistémico de la acción que estudia el comportamiento humano, no es suficiente para explicar por qué la disipación. Las tensiones, los cambios y los conflictos, como tiburones en un estanque, no entran en su formalidad. Este vacío da lugar a la "creación de un nuevo campo" sociológico: la teoría de la acción no lineal y, en general, la teoría de los sistemas abiertos, que encierra el caos, al que se puede aplicar la afirmación de Lorenz al relacionar el aleteo de una mariposa que vuela en la China con un huracán en Texas (Cazau, 2016, p. 68).

En efecto, predicando con el ejemplo, la mariposa movió sus alas en Túnez en 2010 y produjo un huracán en los elementos políticos y sociales de la organización del sistema en el mundo árabe que tomó el nombre de Primavera Árabe. Ese aleteo en Túnez fue una causa pequeña que produjo un gran efecto, el huracán de "la revolución democrática árabe". El hecho instigador, aparentemente intrascendente, ocurrió cuando el buhonero ambulante Mohamed Bouazizi, fue despojado de sus mercancías y sus cuentas de ahorros por parte de la policía y su posterior inmolación, lo cual desató la indignación tunecina en una desproporción tal, que causó un efecto bola de nieve en el sistema total de las tiranías del resto de las naciones árabes: Egipto, Libia, Siria, Yemen, Argelia, Omán, Baréin y Jordania.

Por múltiples razones, pues, la revolución de la Primavera Árabe puede considerarse como tal, según la imagen que de ella se formaron los agentes sociales que propugnaban por un cambio histórico. La acumulación de tensiones en el seno del sistema social entre las tiranías y las expectativas de una juventud educada, pero sin futuro y otros actores, recorrió a tumbos el paisaje político del Magreb en la segunda década del siglo XXI, asimismo que disparó la salida del gobierno autoritario de Ben Ali en Túnez; de Hosni Mubarak, quien llevaba 30 años en el poder, en Egipto; de Muamar Gadafi en Libia; y en Yemen, deponiendo del poder a Ali Abdullah Saleh como al primer ministro Samir Rifai de Jordania, liberando de viejos recuerdos dictatoriales y costumbres políticas engastadas a estos países. 
La teoría determinista está representada por Newton, Laplace y otros pensadores del siglo XVII, cuyas construcciones intelectuales con Descartes, Spinoza, Leibniz, Bacon, Hobbes y Locke, están significadas en los sistemas cerrados. Esta teoría sigue estrictamente el modelo del reloj en la que lo previsible y determinado, forma en efecto, parte de la realidad física. Auguste Comte y Emile Durkheim son dos referentes de esta orientación en la ciencia de lo social en el siglo XIX. Sintetizan el verdadero espíritu del determinismo en el dogma de "la invariabilidad de las leyes físicas", en la fe en los sistemas cerrados y en la necesidad natural que rige la sociedad.

El esquema de la uniformidad del orden de lo real físico de Laplace, plantea que "si conocemos con exactitud las condiciones iniciales del universo, y si conocemos con exactitud las leyes naturales que rigen su evolución, podemos prever exactamente la situación del universo en cualquier instante del tiempo" (Cazau, 2016, p. 67). Esta formulación rigurosa del determinismo científico aparece en el siglo XIX en la sociología positivista.

Para Comte, "la predicción, o la previsión, facilitará el control social (...) el predecir para controlar" (Zeitlin, 1977, p. 91) que es lo propio de un sistema cerrado en el que "lo que es, sigue siendo", reconociendo de las cosas tales como son, es decir, la dignidad de su existencia. La fórmula es, pues, conocer las leyes que rigen la sociedad para prever las posibles vías del futuro social y controlar la vida social, toda vez que se conoce con exactitud las leyes físicas que orientan la situación evolutiva de la sociedad y las condiciones esenciales de su propia existencia.

La física social del siglo XIX considera los fenómenos sociales, según Comte (1999), bajo el doble punto de vista elemental de su armonía con los fenómenos coexistentes y su encadenamiento con el estado anterior (pp. 79, 86). Partiendo de una visión con referencia a la historia de los hechos en los que se ocupa y a su presente histórico, puede predecir las posibles vías del futuro. En suma, el orden de la naturaleza y, por extensión, el orden social, aparece prescrito al determinismo: "si las condiciones no se alteran, un mismo fenómeno se reproducirá indefinidamente, además, siempre es posible determinar el nexo causal que vincula un acontecimiento físico o químico (o social) a las condiciones o acontecimientos anteriores" (Rocher, 1979, p. 27). 
Así, pues, para la doctrina del determinismo científico, el universo funciona como la caída de un cuerpo, no existe lugar para el azar y todo está determinado inexorablemente por las invariables leyes de la naturaleza. Esto hace posible predecir en cualquier situación B, conociendo la anterior situación $\mathrm{A}$, y las leyes naturales que rigen el proceso que va desde $\mathrm{A}$ hasta $\mathrm{B}$.

Según Laplace (1995), "hemos de considerar el estado actual del universo como el efecto de un estado anterior y como la causa del que ha de seguirle" (p. 4). En el campo de la sociología estructuralista, el matrimonio es un buen ejemplo que implica la posibilidad de poder predecir esta relación, entre las reglas matrimoniales en el seno de una población dada y la mutua elección de los cónyuges. La explicación causal de la sucesión de acontecimientos está sujeta al reino del determinismo a la que Durkheim no duda en llamar "la ley de la gravitación del mundo social", por analogía con "la ley de la gravitación universal" de Newton en física.

En el modelo mecánico de la ciencia clásica centrado en la estabilidad, dotado de una razón que hace posible explicar los hechos, al propio tiempo como causas y efectos, y describir el universo como una máquina que funciona de acuerdo con leyes mecánicas que siguen un curso inexorable, el azar es un fenómeno aparente que no forma parte de la multidimensionalidad de la realidad física, es un elemento espurio.

En lo expuesto está contenida la tercera ley de Newton de acción y reacción. En otros términos, si un cuerpo A ejerce una acción sobre otro cuerpo B, este realiza sobre A otra acción igual y de sentido contrario. Esto quiere decir que, un cuerpo no puede cambiar por si solo su estado inicial, ya sea en reposo o en movimiento rectilíneo uniforme, por tanto, se estaría cayendo en una ilusión al considerar que se mueve por sí mismo, a menos que se aplique una fuerza o una serie de fuerzas cuya resultante sea nula. Newton llamó a esta fuerza, gravedad.

Ahora bien, el sistema de las relaciones sostenidas entre una situación consecuente $\mathrm{B}$ y una situación antecedente $\mathrm{A}$, es así examinada desde el punto de vista cualitativo en el mundo newtoniano. La teoría del caos admite que existen vínculos causales, admite la teoría de los vínculos circulares newtonianos, admite la ciencia clásica, pero concibe la injerencia práctica del azar en lo que investiga. 
La sociología no ha asumido una actitud unilateral en el estudio de los hechos colectivos. Tiene tendencias deterministas y tendencias aleatorias, con lo cual, las predicciones y la probabilidad van juntas, pero a su turno, son la "disyuntiva" para el sociólogo según la posición epistemológica que asuma a la luz de las diferentes condiciones históricas, culturales y del clima intelectual de la época.

Cada época de la historia tiene su pensamiento dominante, sea economía clásica, idealismo filosófico o filosofía de la historia. El siglo XIX creyó en las ciencias naturales y en particular, en la física de Newton, en los enormes avances de la física experimental, única forma de análisis verdadera. Así, en estas circunstancias, Newton aparece como el relojero que le da cuerda al sistema de la gravitación universal, lo que influyó profundamente en la elaboración de la física social por Comte para el estudio de la estabilidad de la sociedad con alcances al siglo XX en el análisis de la acción por Parsons. Ha sido Comte quien, en su taller de cadena de montaje, le fijó las piezas del reloj a la física social.

En relación con los hechos de la sociedad, la sociología positiva del siglo XIX tuvo la creencia en la existencia de leyes sociales análogas a las establecidas en la física newtoniana, estando, por tanto, sujeta a la rigidez del determinismo y a las leyes explicativas. La edad contemporánea de la historia, la del siglo XIX, es un hervidero de ideas y doctrinas que se interroga a sí misma permanentemente. En este contexto, la sociedad está sometida a determinismos y a una necesidad natural. En la acera de enfrente, los esfuerzos de la cultura científica, sin embargo, con el materialismo histórico, mezcla el orden con el caos y pone en la guillotina del conocimiento la cabeza del determinismo.

Para Marx, la lucha de clases es el motor instigador, energéticamente significativo, provocador del cambio histórico en la historia de la sociedad mediante un proceso amplificador. La sociedad no es interpretada según leyes naturales. La teoría marxista insinúa que la historia de la sociedad es un ciclo de orden, desorden, orden, que se extiende de la comunidad primitiva hasta hacer su transición al socialismo. Es la constancia de que la lucha de clases lleva el sello a una sociedad no "amarrada a un orden externo y que no piensa ya en sus instituciones como hechos naturales" (Aukon, 1983, p. 160), de ahí que en esa misma vía, por tanto, "la actividad humana (...) 
lleva impresa el sello de la espontaneidad, de la creatividad, de la libertad" (Rocher, 1979, p. 28). Así, pues, las relaciones entre A, lucha de clases y $\mathrm{B}$, transformación social, son vínculos circulares o de feedback, negativos o positivos, en los que las predicciones y el azar coexisten en la realidad social.

Más todavía, el sistema social ha experimentado a través de la historia, una retroalimentación positiva al evolucionar por zonas de incertidumbre hasta llegar al punto de bifurcación, punto en el cual se auto-ordena en una nueva estructura. En la práctica, desde una perspectiva histórica, "cuando se desmorona un régimen colonial para dar lugar a una o varias sociedades nacionales independientes, se producen cambios profundos de estructura, que transforman el antiguo sistema social en otro nuevo, de un tipo diferente" (Rocher, 1979, p. 385).

De hecho, en sentido amplio, la transición de la sociedad tradicional a la sociedad tecnológica, representó cambios estructurales en la organización social, la economía y la mentalidad de estas. Durante el proceso hubo mecanismos de retroalimentación positiva, que lejos de retornar a la economía de subsistencia, la parentela, grupos de edad, o lo sagrado de la sociedad folk, construyó nuevas estructuras económicas, dando lugar a múltiples organizaciones y declarando su fe a la ciencia y el progreso en la sociedad industrial. Así se entiende desde la sociología dispersiva, es decir, la diferencia en el punto de bifurcación entre la retroalimentación negativa y la retroalimentación positiva por el que pasa todo sistema, está en su implicación, o no, en las exigencias del cambio de estructura.

"La teoría del caos, en la medida que considera la existencia de procesos aleatorios", adopta del sistema de relaciones entre causas y efectos, la postura de los procesos independientes, "pero en la medida en que dice que ciertos otros procesos no son caóticos sino ordenados" (Cazau, 2016, p. 65), sostiene que en el sistema de relaciones entre A y B, B y C, entre otras, sí existen vínculos causales.

"Los vínculos causales, por tanto, que más desarrollará son los circuitos de retroalimentación positiva, es decir, aquellos donde se verifica una amplificación de las desviaciones" (Cazau, 2016, p. 65), dicho esto, "una pequeña causa inicial, mediante un proceso amplificador, podrá generar un efecto considerablemente grande" (Cazau, 2016, p. 65), así, por ejemplo, una 
palabra pequeña, pero con gran poder. Esto quiere decir, que la fe tiene la capacidad de mover la mano de Dios. En orden a otros hechos, el "estallido social" en Chile o la "revolución de los treinta pesos" (2019), tuvo como causa instigadora, la modificación, insignificante, en la tarifa del sistema público de transporte de Santiago que provocó una disipación considerable en algunas unidades del sistema total denominada "nueva agenda social".

El modelo no lineal de sistemas abiertos, entra en el estudio científico de los fenómenos colectivos, producidos por el comportamiento humano, generando una tensión fuerte con el modelo mecánico de la ciencia clásica apropiado por la quinta física, como también se conoció a la sociología. El escenario de esta tensión está marcado por el siglo XIX, tal como lo ha expuesto la ciencia de la historia o teoría materialista del caos.

Por lo demás, puede afirmarse, que mucho antes, "los pensadores del siglo XVIII habían perdido la fe en los sistemas metafísicos cerrados y autosuficientes del siglo anterior" (Zeitlin, 1977, p. 4). La ciencia positiva de los hechos del hombre, se va a enfocar en la descripción del universo social y en la explicación de los hechos sociales, según el modelo newtoniano de la física experimental, leído y respetado en alto grado por los positivistas.

Su vocación ha consistido en la conformidad con el concepto de unidad social, demasiado próximo al modelo mecánico. Es la ciencia de la organización y la perpetuación de su unidad por encima de los conflictos y el desplazamiento. La teoría no lineal pone en duda los postulados de la ciencia positiva según el modelo de las ciencias naturales. Toda sociedad constituye un orden precario, a pesar de la armoniosa cooperación entre sus elementos, sujeta a influencias exteriores, energía, comunicación, información, que la llevan a la pérdida del equilibrio en algunas unidades del sistema o de su orientación general.

Varios cientistas sociales han expuesto este concepto de sistema abierto. Los estudios de:

Kroeber acerca de los cambios introducidos por la difusión de los inventos, de las técnicas y de los conocimientos. El minucioso análisis que Bernett hace de diferentes casos de innovación en sociedades arcaicas y en sociedades avanzadas. En el seno mismo de la sociología, Robert Merton, ha analizado 
sobre todo el papel de diferentes factores socioculturales en la evolución de los conocimientos científicos y tecnológicos. Neil Smelser, ha hecho un importante análisis teórico y empírico de los procesos de transformación de la organización y del trabajo industriales, como consecuencia de la introducción progresiva de las máquinas en las empresas textiles inglesas en los siglos XVIII y XIX (Rocher, 1979, p. 404).

Una verdad de simple buen sentido prescribe una cierta proporción entre la causa y el efecto: una fuerza pequeña produce un movimiento pequeño, y una fuerza grande, un gran desplazamiento. En sociología se puede invocar la misma noción para justificar la idea de que una actitud intolerante hacia una pauta de comportamiento desviada, produce cierto tipo de amonestación, y de que una actitud de rechazo e intolerancia permanente genera sanciones importantes y hasta la promulgación de leyes protectoras. En este mismo orden de significaciones, "Francesca Cancian ha indicado cómo el desplazamiento se ha operado en la economía norteamericana entre la propiedad y el control, apoyada en el análisis hecho por Parsons y Smelser” (Rocher, 1979).

Los ejemplos anteriores determinan el modo como los estudios realizados a la sociedad bajo su aspecto histórico de desplazamiento, tienen consecuencias en sus estructuras, quiere decir:

La sociedad es historia (...). La sociedad está incesantemente implicada en un movimiento histórico, en una transformación de sí misma (...). La sociedad suscita, padece o acoge constantemente fuerzas, externas o internas, que modifican su naturaleza, su orientación, su destino (...). Toda sociedad conoce diariamente unos cambios, más o menos acordes con su pasado (...). La sociedad es movimiento y cambio (Rocher, 1979, p. 422).

Ahora bien, ¿̇en qué condiciones? El funcionalismo explica por qué subsisten las sociedades, y no solo el funcionalismo, también el estructuralfuncionalismo desde una sociología del statu quo con T. Parsons, quien muy hipersensible por las nociones de equilibrio en mecánica, explica que la "estabilidad estructural constituye teóricamente un punto de equilibrio entre el sistema social y su entorno, como también en el seno mismo del sistema social” (Rocher, 1979, p. 383), no obstante, no explica por qué 
cambian. Esta afirmación pierde toda validez para la sociología dispersiva, en tanto su discurso sobre las cosas, el universo o la realidad social, es el discurso que estudia la sociedad bajo el aspecto histórico de la bifurcación y la retroalimentación positiva, sin lo cual resulta muy difícil explicar la liquidación de las estructuras de una entidad sistémica como la antigua sociedad agrícola.

¿Qué explicación presenta la sociología dispersiva cuando investiga, por ejemplo, que la antigua sociedad estamentaria no era eterna y que, en consecuencia, era posible una sociedad completamente moderna? ¿Por qué para el funcionalismo y el estructural-funcionalismo el sistema social tiende a perpetuarse tal cual es, a preservar su equilibrio o recuperarlo, cuando las sociedades están en continua ebullición y es difícil expresarlas con nociones que indican un equilibrio con dinámica cero, lo que hace poco probable todo pensamiento de historicidad?

El punto de vista de la sociología dispersiva deja muy en claro que del caos nacen nuevas estructuras, no es destruir "el telar para volver a la rueca", los cambios de la sociedad pueden provenir de causas externas, pero así esté blindada de influencias exteriores, se desplazaría de todos modos al punto de bifurcación hasta constituir una nueva estructura debido al consumo de energía. Así, guiada por su oposición a "la monarquía" de la teoría del orden, hunde sus raíces en "el dadaísmo" de la teoría del caos, que cubre su vida y su actividad.

La sociología aún dispone de los principios de la teoría general de la termodinámica, para el estudio de los actos individuales y grupales asociados a los fines sociales y el cumplimiento del plan evolutivo de la sociedad. Los modelos teóricos opuestos, analizarán la sociedad, como un sistema que no está centrado en la estabilidad, una sociedad con permanentes desequilibrios que la conducen al cambio, a nuevas estructuras y a máquinas no lineales.

Toda sociedad presenta aspectos caóticos, no es una unidad ordenada. La actividad disruptiva de los actos individuales y los conflictos con grupos disidentes, la van caotizando hasta alcanzar el "punto de bifurcación". La ciencia de lo social deja algo muy en claro, que la realidad social no es determinismo absoluto, ni azar permanente, admisión sin reservas que abre las puertas del futuro a la posibilidad de continuar realizando nuevas investigaciones en el campo de la sociología, del caos al orden y del orden al 
caos. El orden y el caos en la sociedad y el orden y el caos en la sociología, son un todo singular, divisible solo analíticamente.

\section{Referencias}

Aukon, A. (1984). La sociología. En Chatelet, F. (Coord.), Historia de la filosofía (Tomo IV) (78-178). Espasa Calpe.

Cazau, P. (2016). La teoría del caos. Fermentum, 26(75), 63-86.

Comte, A. (1999). Discurso sobre el espiritu positivo. Biblioteca Nueva.

Durkheim, E. (1897).Elsuicidio. http://ceiphistorica.com/wp-content/uploads/2016/04/Durkheim -\%C3\%89mile-El-Suicidio.pdf Drucker, P. (1993). La sociedad postcapitalista. Norma.

Laplace, P. S. (1995). Ensayo filosófico sobre las posibilidades. Atalaya.

Rocher, G. (1979). Introducción a la sociología general. Herder.

Zeitlin, I. (1977). Ideología y teoría sociológica. Amorrortu. 